\title{
Application of magnetic resonance diffusion kurtosis imaging for distinguishing histopathologic subtypes and grades of rectal carcinoma
}

\author{
Ziqiang Wen ${ }^{1+}$, Yan Chen ${ }^{1+}$, Xinyue Yang ${ }^{2 \dagger}$, Baolan Lu', Yiyan Liu', Bingqi Shen ${ }^{1}$ and Shenping Yu ${ }^{1 *}$ (D
}

\begin{abstract}
Background: To evaluate the diagnostic performance of diffusion kurtosis imaging (DKI) for distinguishing different histopathological subtypes and grades of rectal carcinoma and to compare DKI with conventional diffusionweighted imaging (DWI).

Methods: This prospective study involved 132 patients with rectal carcinoma, comprising 116 with adenocarcinoma not otherwise specified (AC) and 16 with mucinous carcinoma (MC). High spatial resolution magnetic resonance (MR) and DKI sequences (b values of 0,600, 1000, 1500 and $2000 \mathrm{~s} / \mathrm{mm}^{2}$ ) were performed for pretreatment evaluation. The mean kurtosis (MK) and mean diffusivity (MD) from DKI and the apparent diffusion coefficient (ADC) from DWI were measured by two experienced radiologists. The Mann-Whitney $U$ test was used to evaluate different histopathological subtypes and grades. Receiver operating characteristic (ROC) curve analyses were performed to compare the diagnostic ability of different quantitative parameters.

Results: The MD and ADC values were significantly higher for MC than for AC (1.94 \pm 0.51 vs. $1.33 \pm 0.02$ and $1.26 \pm 0.64$ vs. $0.92 \pm 0.01$, respectively; $P<0.001)$. The MK values were significantly lower for $M C$ than for $A C(0.66 \pm 0.02$ vs. $0.93 \pm 0.09, P<0.001)$. The MK and MD values demonstrated higher sensitivity $(94 \%$, both) and specificity (96, 93\%, respectively) than the ADC values. However, all the parameters derived from both DKI and DWI showed no significant differences between different histological grades.

Conclusions: DKI is a more valuable imaging biomarker than conventional DWI for differentiating MC from AC. However, it is still debatable whether DKI is useful for distinguishing different histological grades.
\end{abstract}

Keywords: Rectal cancer, Magnetic resonance imaging, Diffusion kurtosis imaging, Mucinous carcinoma, Imaging biomarker

\section{Background}

Rectal carcinoma is one of the most common digestive malignant tumors worldwide [1], and for the past several years, evidence has revealed that the popularity of screening and changes in treatment strategies have delayed the progression of disease and decreased the overall mortality rates of patients with rectal carcinoma [1]. Previously, studies

\footnotetext{
* Correspondence: yushp@mail.sysu.edu.cn

${ }^{\dagger}$ Ziqiang Wen, Yan Chen and Xinyue Yang contributed equally to this work. ${ }^{1}$ Department of Radiology, Sun Yat-sen University First Affiliated Hospital, No. 58, Zhongshan Second Road, Yuexiu District, Guangzhou 510080, China Full list of author information is available at the end of the article
}

indicated that preoperative neoadjuvant therapy was effective for decreasing local recurrence and increasing the chance of enhancing sphincter preservation compared with surgery alone $[2,3]$. However, the response to treatment in rectal carcinoma is associated with the histopathological subtype and histological grade of disease $[4,5]$. In particular, mucinous carcinoma (MC) does not respond well to neoadjuvant therapy and has a poorer prognosis than other types of rectal carcinoma [6]. In addition, side effects from preoperative chemoradiotherapy are not uncommon [7]. Therefore, it is of considerable importance to assess related

(c) The Author(s). 2019 Open Access This article is distributed under the terms of the Creative Commons Attribution 4.0 International License (http://creativecommons.org/licenses/by/4.0/), which permits unrestricted use, distribution, and reproduction in any medium, provided you give appropriate credit to the original author(s) and the source, provide a link to the Creative Commons license, and indicate if changes were made. The Creative Commons Public Domain Dedication waiver (http://creativecommons.org/publicdomain/zero/1.0/) applies to the data made available in this article, unless otherwise stated. 
factors to determine individual treatment strategies and prevent side effects from unnecessary therapy.

High spatial resolution magnetic resonance (MR) imaging has been routinely used for the preoperative evaluation of rectal carcinoma [8-10]. In addition, functional MR imaging, such as diffusion-weighted imaging (DWI), has been widely regarded as a promising tool for preoperative assessment of rectal carcinoma. This imaging modality can provide information on a molecular level in addition to anatomical information [11-14]. However, conventional DWI assumes that the motion of water molecules distributes according to Gaussian behavior. Notably, the microenvironment in biological tissue is complex [15]. Water molecule diffusion with non-Gaussian behavior results from microstructures such as the cytomembrane and extracellular matrix and contributes to diffusion. Jensen et al. proposed a non-Gaussian diffusion model called diffusion kurtosis imaging (DKI), which includes mean kurtosis (MK) and mean diffusivity (MD), to quantify this deviation from Gaussian form [16]. In previous studies, DKI exhibited advantages for glioma, breast cancer and masses located in the head and neck region [17-20]. To our knowledge, there are few studies on DKI for differentiating histopathological subtypes and grades of rectal carcinoma, especially for MC.

Therefore, our study was aimed at proving the applicability of DKI in rectal carcinoma. We compared the features of adenocarcinoma not otherwise specified (AC), which was the most common form of rectal carcinoma and other special subtypes were excluded, and unaffected rectal wall tissues first and further compared parameters from DKI and DWI between different histologic subtypes and grades of rectal carcinoma.

\section{Methods}

\section{Patients}

This prospective study was approved by our institutional ethics review board, and informed consent was obtained from all patients. The inclusion criteria were as follows: (1) colonoscopy with confirmed primary rectal carcinoma by biopsy; (2) routine high-resolution MR and DKI sequences; (3) MR examination performed three days or more after biopsy; and (4) surgery performed approximately 2-7 days after MR examination. So 148 patients were included in the study at the first. 16 patients were excluded for satisfying one of the following exclusion criteria: (1) pathological diagnosis other than rectal carcinoma; (2) neoadjuvant therapy administered before MR examination; (3) insufficiently large parenchymal area in the tumor to select regions of interest (ROIs); and (4) unsatisfactory image quality with serious artifact. Eventually 132 patients including 116 patients (median age, 52 years; range, $29-83$ years; 70 men and 46 women) with $\mathrm{AC}$ and 16 patients (median age, 51 years; range, 22-67 years; 8 men and 8 women) with MC were enrolled in this prospective study (Fig. 1).

\section{MR examination}

The patients in this study all underwent MR examinations with a 3.0-T MR scanner (Magnetom Verio, Siemens Healthcare, Erlangen, Germany) with a six-channel body matrix coil. Prior to the MR scan, $20-80 \mathrm{~mL}$ of ultrasound gel was injected into the rectum to display the boundaries of the tumor more clearly. The quantity of the gel injected into the rectum in each patient depended on the distance from the inferior part of the tumor to the anal verge. Each patient received a routine intramuscular injection of $20 \mathrm{mg}$ of anisodamine to reduce artifacts caused by peristalsis of the intestinal tract. Gadolinium (Gadopentetate Dimeglumine Injection, Consun, Guangzhou, China) was intravenously injected using a power injector (Medrad, Pittsburgh, PA, USA) at $0.2 \mathrm{~mL} / \mathrm{kg}$ of body weight at a rate of $3.0 \mathrm{~mL} / \mathrm{s}$.

Conventional rectal MR imaging included the following sequences: (1) an axial T2-weighted turbo spin-echo sequence with thick sections; (2) a high-resolution sagittal T2-weighted turbo spin-echo sequence with thin sections in sagittal, coronal and oblique axial planes.

A DKI sequence was performed in an axial plane through the use of a single-shot echo-planar imaging sequence with b values of $0,600,1000,1500$ and $2000 \mathrm{~s} / \mathrm{mm}^{2}$ (Table 1).

\section{Imaging analysis}

All primary data of 132 patients were uploaded to a workstation, and parameter maps of DKI were generated by using postprocessing software that was developed in house (MATLAB Version 2.1; MathWorks, Natick, MA, USA). For the DWI dataset, imaging data of $2 \mathrm{~b}$ values $\left(0,1000 \mathrm{~s} / \mathrm{mm}^{2}\right)$ were processed to calculate ADC maps. Data of $5 \mathrm{~b}$ values $\left(0,600,1000,1500\right.$ and $\left.2000 \mathrm{~s} / \mathrm{mm}^{2}\right)$ were processed using a three-variable linear leastsquares method based on a DKI model similar to that implemented in a previous study to get $\mathrm{MK}$ and MD maps [21].The linear fitting equation is as follows:

$$
\ln \left(S_{b}\right)=\ln \left(S_{0}\right)-\mathrm{b} \cdot \mathrm{D}+1 / 6 \cdot b^{2} \cdot D^{2} \cdot K
$$

where $S_{b}$ indicates the MR signal intensity at the particular $\mathrm{b}$ value used; $\mathrm{S}_{0}$ indicates the MR signal intensity when there is no diffusion gradient; $\mathrm{K}$ is the apparent diffusional kurtosis, which is a dimensionless metric of departure from Gaussian behavior of water motion; and $\mathrm{D}$ is the apparent diffusion coefficient (ADC), which has been revised for non-Gaussian behavior.

The patients' MR data were reviewed by two seasoned radiologists who were blinded to the pathological and clinical information. The three largest sections of tumor parenchyma and three sections of unaffected rectal wall that were located more than 1 $\mathrm{cm}$ from the tumor were manually drawn on high-b-value images and later were copied that to all DWI and DKI derived maps (Figs. 2, 3). The ROIs 
Inclusion criteria:

(1) colonoscopy with confirmed primary rectal carcinoma by biopsy;

(2) routine high-resolution MR and DKI sequences;

(3) MR examination performed three days or more after biopsy;

(4) surgery performed approximately 2-7 days after MR examination

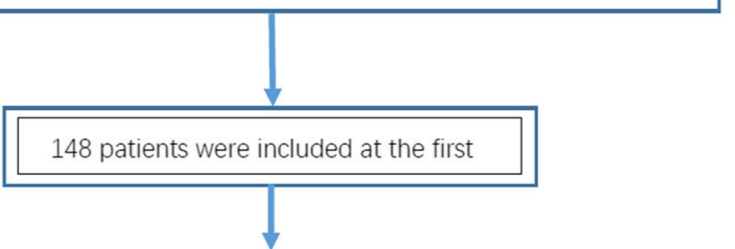

Exclusion criteria:

(1) pathological diagnosis other than rectal carcinoma( $n=1)$;

(2) neoadjuvant therapy administered before MR examination( $n=5)$;

(3) insufficiently large parenchymal area in the tumor to select regions of interest $(n=8)$;

(4) unsatisfactory image quality with serious artifact $(n=2)$

\section{2 patients were eventually enrolled}

Fig. 1 Flowchart of the study population

Table 1 MR Imaging Protocol Parameters for T2-Weighted Imaging and DKI

\begin{tabular}{|c|c|c|c|c|c|}
\hline \multirow[b]{4}{*}{ Parameter } & \multicolumn{5}{|c|}{ Imaging Sequence } \\
\hline & & Sagittal & Coronal & Oblique Axial & Axial \\
\hline & Axial & High-resolution & High-resolution & High-resolution & Diffusion- \\
\hline & $\mathrm{T} 2 \mathrm{~W}$ & $\mathrm{~T} 2 \mathrm{~W}$ & $\mathrm{~T} 2 \mathrm{~W}$ & $\mathrm{~T} 2 \mathrm{~W}$ & kurtosis \\
\hline Pulse sequence & TSE & TSE & TSE & TSE & DKI-EPI \\
\hline TR/TE (ms) & $3000 / 87$ & $3000 / 87$ & $4000 / 77$ & $3000 / 84$ & $3800 / 74.7$ \\
\hline Echo train length & 14 & 12 & 21 & 16 & / \\
\hline $\mathrm{FOV}\left(\mathrm{mm}^{2}\right)$ & $260 \times 260$ & $180 \times 180$ & $220 \times 220$ & $180 \times 180$ & $300 \times 245$ \\
\hline Section thickness $(\mathrm{mm})$ & 5 & 3 & 3 & 3 & 6 \\
\hline No. of slices & 25 & 19 & 25 & 24 & 21 \\
\hline Dist. factor & $20 \%$ & 0 & 0 & 0 & $20 \%$ \\
\hline Phase oversampling & $70 \%$ & $70 \%$ & $100 \%$ & $70 \%$ & $20 \%$ \\
\hline Voxel size $\left(\mathrm{mm}^{3}\right)$ & $0.8 \times 0.7 \times 5.0$ & $0.7 \times 0.6 \times 3.0$ & $0.7 \times 0.6 \times 3.0$ & $0.7 \times 0.6 \times 3.0$ & $2.7 \times 2.7 \times 6.0$ \\
\hline Averages & 2 & 2 & 2 & 2 & 2 \\
\hline Concatenation & 2 & 2 & 1 & 2 & 1 \\
\hline Acquisition time & $2 \min 56 s$ & $2 \mathrm{~min} 30 \mathrm{~s}$ & $2 \min 52 \mathrm{~s}$ & $3 \min 18 s$ & $6 \min 1 \mathrm{~s}$ \\
\hline
\end{tabular}

Note: $D K I=$ diffusion kurtosis imaging, $E P I=$ echo-planar imaging, $T S E=$ turbo spin-echo, $\mathrm{T} 2 \mathrm{~W}=\mathrm{T} 2$-weighted, $\mathrm{TR}=$ repetition time, $\mathrm{TE}=\mathrm{echo}$ time, $\mathrm{FOV}=$ field of view 

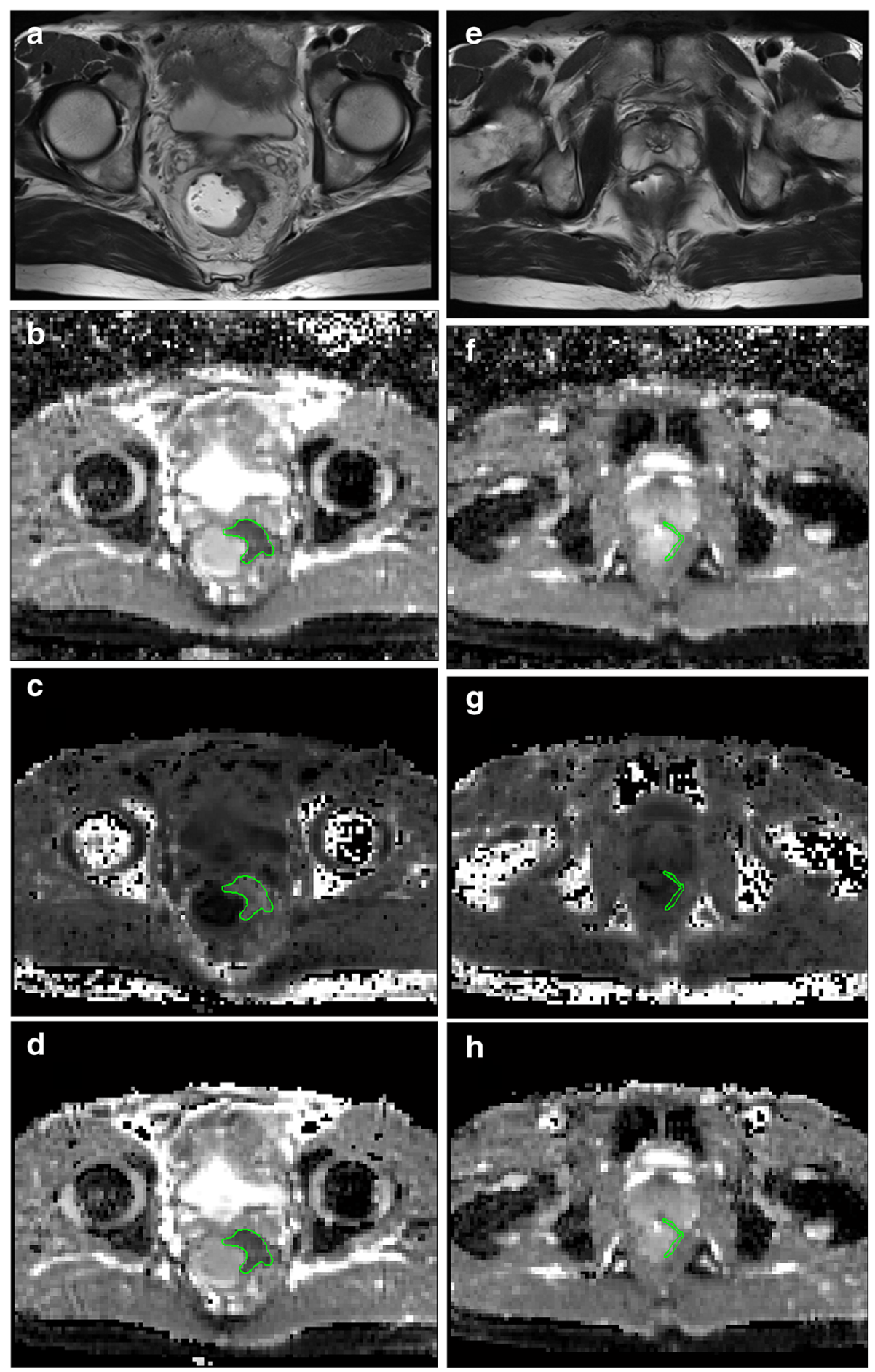

Fig. 2 Images above show Grade 1 AC and unaffected rectal wall in a 62-year-old man. Axial T2-weighted MR image shows the AC along the left wall of the rectum (a). ADC map shows low-signal-intensity tumor ( $\mathrm{ADC}=1.09 \pm 0.24 \times 10^{-3} \mathrm{~mm}^{2} / \mathrm{s}, \mathbf{b}$ ). MK map shows high-signal-intensity tumor ( $M K=1.00 \pm 0.19, \mathbf{c})$. MD map shows low-signal-intensity tumor $\left(M D=1.26 \pm 0.24 \times 10^{-3} \mathrm{~mm}^{2} / \mathrm{s}\right.$, d). Axial T2-weighted MR image shows the unaffected rectal wall (e). ADC map shows high-signal-intensity rectal wall ( $A D C=1.69 \pm 0.10 \times 18^{-3} \mathrm{~mm}^{2} / \mathrm{s}$, $\mathbf{f}$ ). MK map shows low-signalintensity rectal wall $(M K=0.57 \pm 0.10, \mathbf{g})$. MD map shows high-signal-intensity rectal wall $\left(\mathrm{MD}=2.04 \pm 0.21 \times 10^{-3} \mathrm{~mm}^{2} / \mathrm{s}, \mathrm{h}\right)$ 

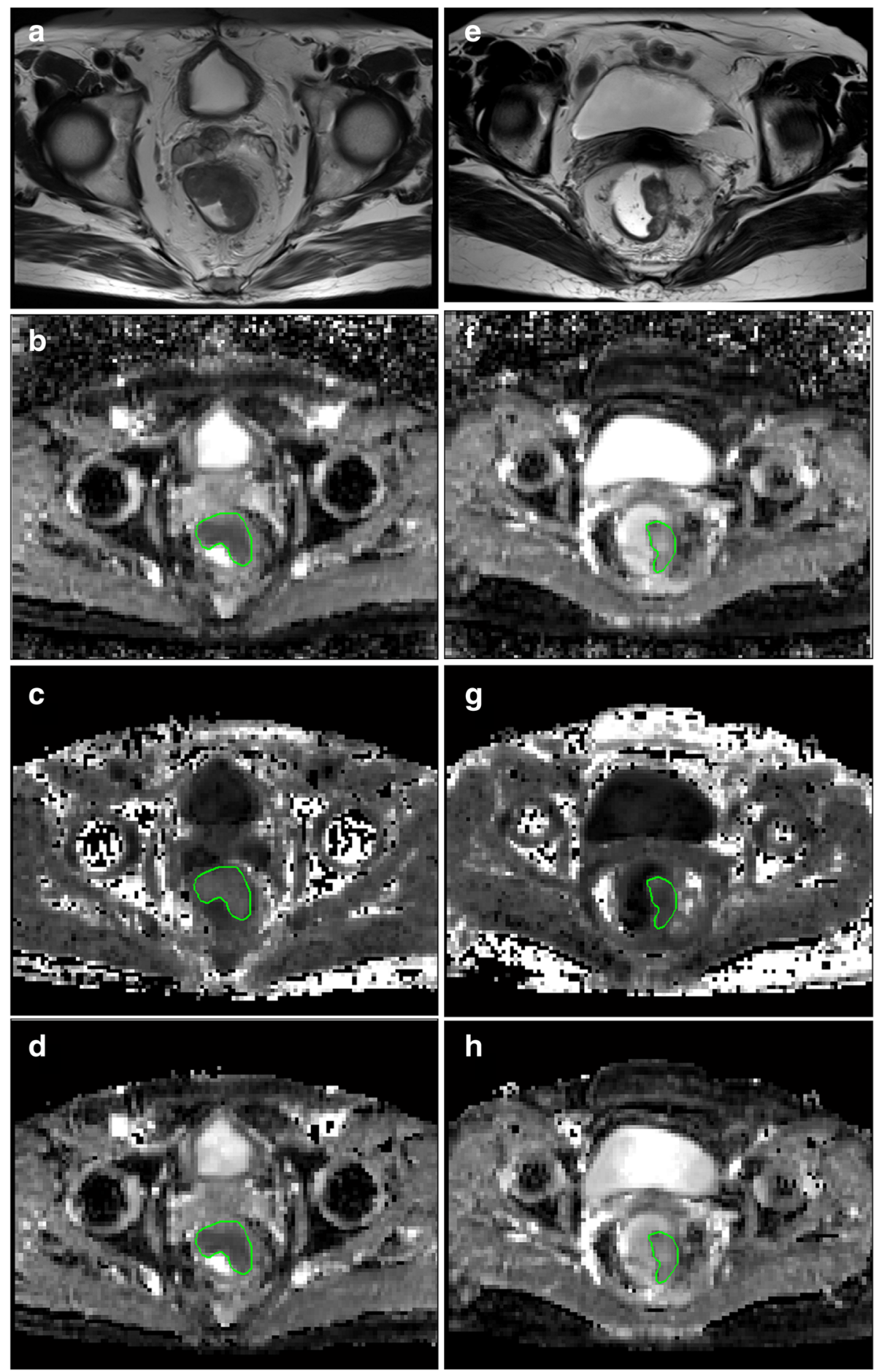

Fig. 3 Images above show a 54-year-old man with Grade 2 AC (a-d) and a 72-year-old woman with Grade 3 MC (e-h). Axial T2-weighted MR image shows the $A C$ along the anterior and left wall of the rectum (a). ADC map shows low-signal-intensity tumor $\left(A D C=1.21 \pm 0.92 \times 10^{-3}\right.$ $\left.\mathrm{mm}^{2} / \mathrm{s}, \mathbf{b}\right)$. MK map shows high-signal-intensity tumor ( $\left.M K=1.02 \pm 0.01, \mathbf{c}\right)$. MD map shows low-signal-intensity tumor $\left(\mathrm{MD}=1.19 \pm 0.21 \times 10^{-3}\right.$ $\mathrm{mm}^{2} / \mathrm{s}$, d). Axial T2-weighted MR image shows the MC along left wall of the rectum (e). ADC map shows high-signal-intensity tumor (ADC=1.84 $\pm 0.57 \times 10^{-3} \mathrm{~mm}^{2} / \mathrm{s}$, f). MK map shows low-signal-intensity tumor (MK $\left.=0.72 \pm 0.15, \mathbf{g}\right)$. MD map shows high-signal-intensity tumor $\left(\mathrm{MD}=1.88 \pm 0.31 \times 10^{-3} \mathrm{~mm}^{2} / \mathrm{s}, \mathrm{h}\right)$ 
were confirmed by comparing the ROI position in the parameter maps to axial T2-weighted imaging. Areas of necrosis, cystic degeneration and intestinal contents were avoided. The pixel-based mean value of the three sections represented the value of the tumor and unaffected rectal wall, which were averaged for comparison. The values measured from radiologist 1 were used in comparison.

\section{Pathological assessment}

All the surgical specimens were routinely processed by the pathology department with fixation in formalin and stained with hematoxylin-eosin. Pathological evaluation was performed by pathologists on the basis of the seventh edition of the American Joint Committee on Cancer's TNM classification [22]. Tumors were classified as Grade 1 (well differentiated) when gland-like structures accounted for more than $95 \%$ of the specimen, Grade 2 (moderately differentiated) when these structures occupied more than $50 \%$ but less than or equal to $95 \%$ of the specimen, or Grade 3 (poorly differentiated) when these structures occupied less than or equal to $50 \%$ of the specimen. $\mathrm{MC}$ was characterized by abundant extracellular mucin that constituted more than $50 \%$ of the tumor volume [22].

\section{Statistical analysis}

The numeric data of DKI and DWI parameters measured by the two radiologists were divided into different groups. The mean and standard deviation were calculated for each parameter. The Kolmogorov-Smirnov test was used to perform a normality analysis on the data of the different groups. The data were not normally distributed. So the Wilcoxon test was used to compare the differences in each parameter in the following groups: AC and unaffected rectal wall. The differences in each parameter in these groups (MC vs. AC; Grade 1-2 vs. Grade 3) were assessed by using the Mann-Whitney $U$ test. Receiver operating characteristic (ROC) curve analysis was performed to assess the diagnostic performance of each parameter. Sensitivity and specificity were measured with a threshold determined by using the maximum Youden's index. Area under the curves (AUC) were compared using the method developed by Delong et al. [23]

Interreader agreement for DKI and DWI derived parameter measurements were estimated by using interclass correlation coefficient (ICC) and were interpreted as follows: ICC $>0.75$ indicated excellent agreement, 0.60-0.75 indicated good agreement, $0.40-0.60$ indicated fair agreement, and less than 0.40 indicated poor agreement. Statistical analysis was performed with SPSS (version 23.0) and MedCalc (version 15.8) software. $P$ values $<0.05$ were considered statistically significant.

\section{Results}

\section{Patient demographics and histological results}

In total, 132 patients satisfied the study criteria mentioned above, and ultimately 116 patients (70 men and 46 women; median age: 52 years; age range: $29-83$ years) with $\mathrm{AC}$ and 16 patients ( 8 men and 8 women; median age: 51 years; age range: $22-67$ years) with $\mathrm{MC}$ were enrolled.

The pathological evaluation by using the WHO grading criteria was as follows: AC (Grade 1: $n=1$; Grade 2: $n=93$; Grade 3: $n=22$ ) and MC (Grade 1: $n=1$; Grade 2: $\mathrm{n}=9$; Grade 3: $n=6$ ). The correlative pathological and clinical characteristics are listed in Table 2.

\section{Interobserver agreement}

The interobserver agreement between the two radiologists was assessed by the ICC (Table 3). There was excellent agreement between the two observers for $\mathrm{MK}, \mathrm{MD}$, and ADC.

\section{Comparison between $\mathrm{AC}$ and unaffected rectal wall}

The MD and ADC values were significantly lower in AC than in unaffected rectal wall $(1.33 \pm 0.02$ vs. $2.20 \pm 0.34$, $P<0.001$ for $\mathrm{MD} ; 0.92 \pm 0.01$ vs. $1.59 \pm 0.03, P<0.001$ for $\mathrm{ADC})$. The $\mathrm{MK}$ values for $\mathrm{AC}$ were significantly

Table 2 Clinical and Pathological Characteristics

\begin{tabular}{lll}
\hline & AC (116) & MC (16) \\
\hline Age & 52 & 51 \\
Median age & $29-83$ & $22-67$ \\
Age range & & \\
Gender & 70 & 8 \\
Male & 46 & 8 \\
Female & & \\
Histological Grade & 1 & 1 \\
Grade 1 & 93 & 9 \\
Grade 2 & 22 & 6 \\
Grade 3 & & \\
MR T Stage & 0 & 0 \\
T1 & 17 & 2 \\
T2 & 59 & 9 \\
T3 & 40 & 5 \\
T4 &
\end{tabular}

Note: $A C=$ adenocarcinoma not otherwise specified, $M C=$ mucinous carcinoma 
Table 3 ICC for the Parameters Measured by Two Observers

\begin{tabular}{llll}
\hline Parameters & MK & MD & ADC \\
\hline ICC $(95 \%$ Cl) & $0.96(0.93,0.97)$ & $0.97(0.96,0.98)$ & $0.78(0.69,0.85)$ \\
\hline
\end{tabular}

Note: Data in parentheses are $95 \%$ confidence intervals, ICC = interclass correlation coefficient, $M K=$ mean kurtosis, $M D=$ mean diffusivity $A D C=$ apparent diffusion coefficient

higher than those for unaffected rectal wall $(0.93 \pm 0.09$ vs. $0.59 \pm 0.12, P<0.001$ ) (Table 4 ) (Fig. 4a).

\section{Performance of $M K, M D$ and $A D C$ for distinguishing MC from $A C$}

The MD and ADC values were significantly higher in $\mathrm{MC}$ than in $\mathrm{AC}(1.94 \pm 0.51$ vs. $1.33 \pm 0.02, P<0.001$ for MD; $1.26 \pm 0.64$ vs. $0.92 \pm 0.01, P<0.001$ for ADC). The MK values were significantly lower in $\mathrm{MC}$ than in $\mathrm{AC}$ $(0.66 \pm 0.02$ vs. $0.93 \pm 0.09, P<0.001)$ (Table 4$)$ (Fig. $4 \mathrm{~b}$ ). ROC analysis demonstrated that MK showed the highest sensitivity (94\%) and specificity (96\%) with a cut-off value of 0.75 (Table 5). Comparisons of the ROC curves of these parameters indicated that MK had the highest area under the curve (AUC) of 0.97 . There was no significant difference between the AUC derived from DKI and conventional DWI. However, the AUC of MK and MD were higher than that of ADC substantially (Fig. 5).

\section{Performance of $M K, M D$ and $A D C$ for distinguishing grade} 1-2 AC from grade $3 \mathrm{AC}$

All the parameters showed no significant difference between Grade 1-2 and Grade 3 AC (Table 4) (Fig. 4c).

\section{Discussion}

In our study, all the parameters derived from DKI and conventional DWI showed significant differences between $\mathrm{AC}$ and unaffected rectal wall. In addition, our study revealed another important finding; namely, MC showed higher MD and $\mathrm{ADC}$ values than those of $\mathrm{AC}$. The MK values in MC were lower than those in AC.
However, all the parameters were not different between Grade 1-2 AC and Grade 3 AC.

In agreement with previous study [24], the ADC values for unaffected rectal wall were significantly higher than those for AC. For the DKI derived parameters, the MD values for unaffected rectal wall were significantly higher than those for $\mathrm{AC}$ while the MK values were significantly lower for unaffected rectal wall. These differences in the parameters obtained from conventional DWI and DKI in AC may indicate the clinical feasibility of DKI for evaluating rectal carcinoma. The high cellularity and decreased extracellular space in these tumors may cause restriction of water molecular motion [25], which can explain the differences between tumor and unaffected rectal wall tissues.

MC is distinguished as a totally different subtype of colorectal carcinoma with a poor prognosis and poor response to preoperative chemoradiotherapy [6]. Accurate diagnosis of this subtype of rectal carcinoma is significant for determining an appropriate treatment plan. Yu et al. [26] found that preoperative MR imaging was a more accurate method for detecting $\mathrm{MC}$ than biopsy sampling. However, the T2-weighted MR evaluation is still a subjective method because its diagnostic accuracy is correlated with the experience of the interpreting radiologists. Thus, we hope to use functional MR imaging techniques, such as DKI and DWI, to quantitatively distinguish $\mathrm{MC}$ from $\mathrm{AC}$. In our study, the MD and ADC values for MC were higher than those for $\mathrm{AC}$; these findings are in agreement with those of Nasu et al. [27]. We assumed that these differences might be associated with the amount of extracellular mucus in mucinous rectal carcinoma, which causes low cellularity, while the higher cellularity in $\mathrm{AC}$ might further restrict water molecule movement in tumor tissue. On the other hand, our study indicated that the MK values were significantly lower in $\mathrm{MC}$ than in $\mathrm{AC}$. As an extension of conventional DWI, DKI can reflect tissue heterogeneity [16]. Because of its high cellularity and various membranes,

Table 4 MK, MD and ADC values Among Different Groups

\begin{tabular}{lllllll}
\hline & AC & Unaffected wall & MC & AC & Grade 1-2 AC & Grade 3 AC \\
\hline Number & 116 & 116 & 16 & 116 & 94 & 22 \\
MK & $0.93 \pm 0.09$ & $0.59 \pm 0.12$ & $0.66 \pm 0.02$ & $0.93 \pm 0.09$ & $0.94 \pm 0.08$ & $0.92 \pm 0.12$ \\
$P$ & $<0.001$ & & $<0.001$ & & 0.22 & $1.28 \pm 0.14$ \\
MD & $1.33 \pm 0.02$ & $2.20 \pm 0.34$ & $1.94 \pm 0.51$ & $1.33 \pm 0.02$ & 0.97 & $0.28 \pm 0.18$ \\
$P$ & $<0.001$ & & $<0.001$ & & $0.91 \pm 0.83$ & $0.90 \pm 0.12$ \\
ADC & $0.92 \pm 0.01$ & $1.59 \pm 0.03$ & $1.26 \pm 0.64$ & $0.92 \pm 0.01$ & & 0.90 \\
$P$ & $<0.001$ & & $<0.001$ & & & \\
\hline
\end{tabular}

Note: Data are means \pm standard deviations, $M D$ and $A D C$ values are given in $\mathrm{mm}^{2} / \mathrm{s} \times 10^{-3}, A C=$ adenocarcinoma not otherwise specified, $M C=$ mucinous carcinoma, $\mathrm{MK}=$ mean kurtosis, $\mathrm{MD}=$ mean diffusivity 


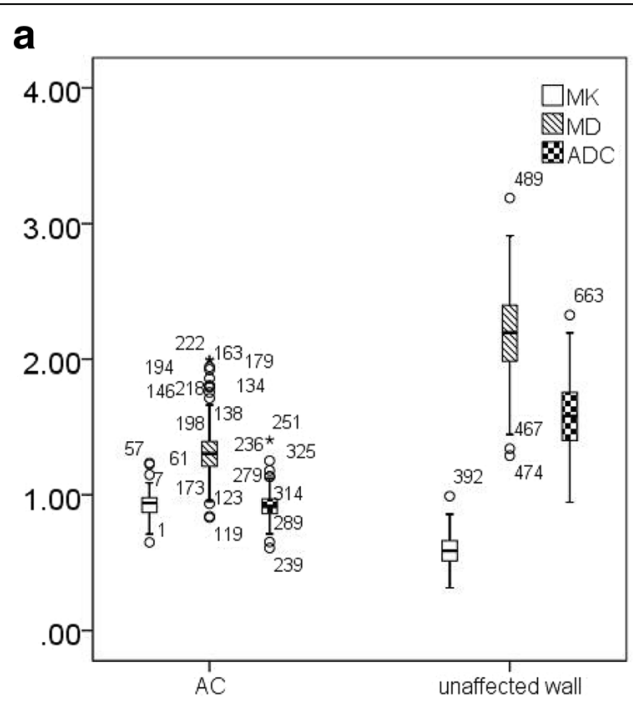

b

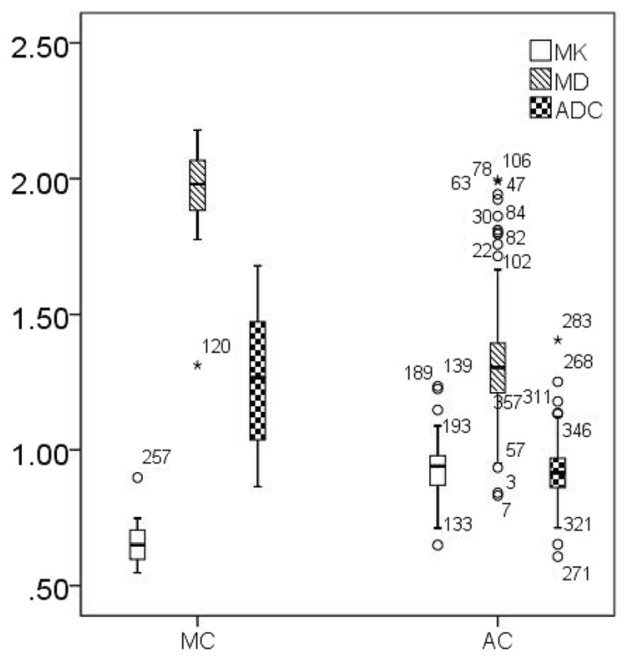

C

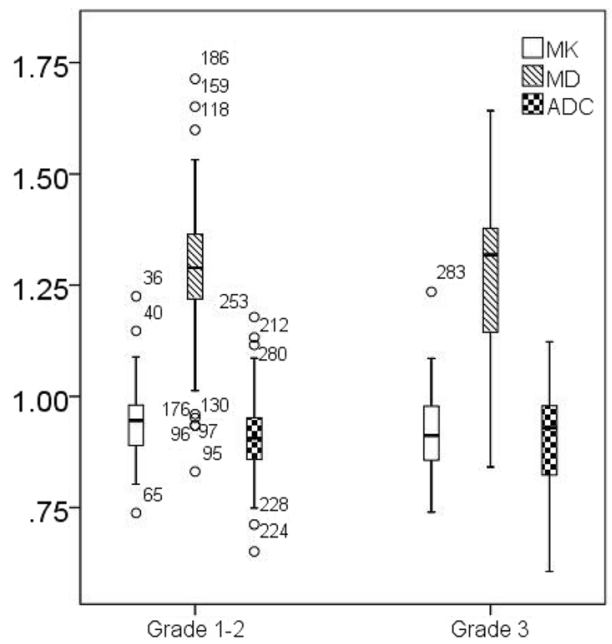

Fig. 4 Box plots showing comparison of $M K, M D$ and $A D C$ among different groups (a, AC vs. unaffected wall; b, MC vs. AC; $\mathbf{c}$, Grade 1-2 AC vs. Grade 3 AC)
Table 5 Receiver Operating Characteristic Analysis of MK, MD and ADC

\begin{tabular}{llll}
\hline Parameters & MK & MD & ADC \\
\hline AUC & 0.97 & 0.95 & 0.88 \\
$P_{\text {AUC }}$ & MK vs. MD & MK vs. ADC & MD vs. ADC \\
& 0.64 & 0.15 & 0.24 \\
Cut-Off Value & $\leq 0.75$ & $>1.76$ & $>1.06$ \\
Sensitivity (100\%) & 0.94 & 0.94 & 0.75 \\
Specificity (100\%) & 0.96 & 0.93 & 0.92 \\
\hline Note: AUC = area under & ROC curve, $R O C=$ receiver operating characteristic
\end{tabular}

AC may lead to more deviation from Gaussian behavior. In addition, the results of the ROC analysis demonstrated that using the MK and MD values obtained from DKI might have higher specificity and sensitivity for distinguishing $\mathrm{MC}$ from $\mathrm{AC}$, suggesting better diagnostic performance of DKI than conventional DWI. Similar findings have been reported in a previous study that showed the advantages of DKI in different diseases [18-20, 28]. These findings suggest that DKI can offer additional information regarding changes in microstructure in different tumor tissues.

Our study also suggested that there were no significant differences in any of the parameters for distinguishing Grade 1-2 and Grade 3 AC. Similar results have been observed in some previous studies [24]. However, our results were contrary to those of another study by Zhu L et al. [21]. We hypothesize that this difference might be caused by different selection of $b$ values between the two studies because the number of $b$ values used and the actual $b$ values are crucial for optimal fitting DKI model [24]. On the other hand, the sample sizes might also have been an important factor that impacted the results. In our study, the number of Grade $1 \mathrm{AC}$ was relatively few, which might have caused a bias. Therefore, the application of DKI and DWI for pathologic grading of rectal carcinoma remains debatable.

Additionally, the ICC of ADC values was lower substantially than that of $\mathrm{MK}$ and MD values relatively, which was incomprehensible because they were measured from the same ROI. However, similar finding also can be found in other previous study. According to Lambregts et al. [29] research, we assumed that single-slice evaluation might be one of the reason for this different ICC while whole-volume evaluation might provide more reproducible results.

Our study has several limitations. First, this was a single-center study, and this sample size was not large enough, especially for Grade 1 rectal carcinoma, which might have resulted in bias in the analysis of different grade groups. Thus, a multicenter study of a large cohort is necessary. Furthermore, single-slice ROI analysis might not have accurately reflected the 


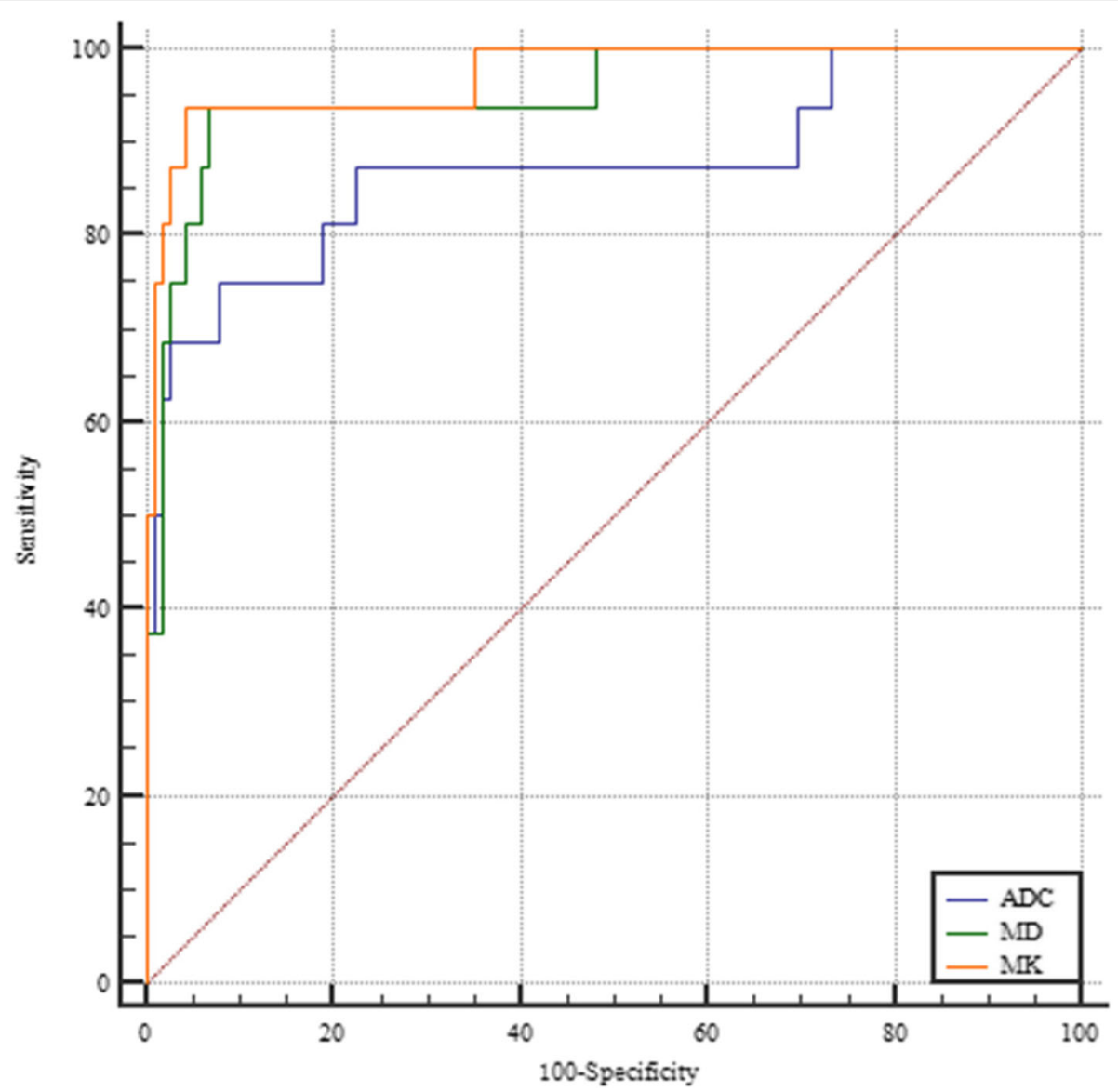

Fig. 5 ROC curves of performance of $M K, M D$ and $A D C$ for discriminating $M C$ from $A C$

characteristics of the whole tumor parenchyma. Histogram analysis could give more comprehensive information. Finally, injection of ultrasound gel into the rectum might have thinned the unaffected wall and increased the measurement error. A controlled test without injecting gel should be performed to determine the effect of this bias.

\section{Conclusions}

DKI is a potentially promising technique for distinguishing $\mathrm{MC}$ from $\mathrm{AC}$. Furthermore, parameters derived from DKI performed better than those derived from conventional DWI for differentiating between subtypes of rectal carcinoma. However, it is still debatable whether DKI or DWI can distinguish between different histopathological grades of rectal carcinoma.

\section{Abbreviations}

AC: Adenocarcinoma not otherwise specified; ADC: Apparent diffusion coefficient; AUC: Area under curve; Cl: Confidence interval; DKI: Diffusion kurtosis imaging; DWI: Diffusion-weighted imaging; ICC: Interclass correlation coefficient; MC: Mucinous carcinoma; MD: Mean diffusivity; MK: Mean kurtosis; MR: Magnetic resonance; ROC: Receiver operating characteristic; ROI: Region of interest; TA: Acquisition time; TE: Echo time; TR: Repetition time

\section{Acknowledgements}

We hold the honor to get technical support from Xu Yan, PhD, a collaboration scientist from Siemens Healthcare.

\section{Funding}

The funding source was province science and technology of Guangdong (No. 2014A020212126).

Availability of data and materials

The datasets supporting the conclusion of this article available from the corresponding author on reasonable request.

\section{Authors' contributions}

SY put forward the study concepts and ZW designed the study. ZW and YC made contribution to collecting patients. ZW and XY made statistical analysis. BL, YL and BS made data analysis and interpretation. ZW, YC and XY were major contributors and contributed equally in writing the manuscript. All authors made a substantial contribution to researching data, discussion of content, reviewing and editing manuscript before submission. All authors read and approved the final manuscript.

\section{Ethics approval and consent to participate}

This study was approved by our institutional ethics committee.

\section{Consent for publication}

Written informed consent was obtained from each patient.

\section{Competing interests}

The authors declare no conflict of interest to disclose. 


\section{Publisher's Note}

Springer Nature remains neutral with regard to jurisdictional claims in published maps and institutional affiliations.

\section{Author details}

'Department of Radiology, Sun Yat-sen University First Affiliated Hospital, No. 58, Zhongshan Second Road, Yuexiu District, Guangzhou 510080, China. ${ }^{2}$ Department of Radiology, Southern Medical University Zhujiang Hospital, Guangzhou 510282, China.

Received: 8 August 2018 Accepted: 28 January 2019

Published online: 11 February 2019

\section{References}

1. Siegel RL, Miller KD, Fedewa SA, Ahnen DJ, Meester RGS, Barzi A, Jemal A. Colorectal cancer statistics, 2017. CA Cancer J Clin. 2017;67(3):177-93.

2. Bosset JF, Collette L, Calais G, Mineur L, Maingon P, Radosevicjelic L, Daban A, Bardet E, Beny A, Ollier JC. Chemotherapy with preoperative radiotherapy in rectal cancer. N Engl J Med. 2006;355(11):1114-23.

3. Sebagmontefiore D, Stephens RJ, Steele R, Monson J, Grieve R, Khanna S, Quirke P, Couture J, Metz CD, Myint AS. Preoperative radiotherapy versus selective postoperative chemoradiotherapy in patients with rectal cancer (MRC CR07 and NCIC-CTG C016): a multicentre, randomised trial. Lancet. 2009:373(9666):811.

4. Mccawley N, Clancy C, O'Neill BD, Deasy J, Mcnamara DA, Burke JP. Mucinous rectal adenocarcinoma is associated with a poor response to neoadjuvant Chemoradiotherapy: a systematic review and meta-analysis. Dis Colon Rectum. 2016:59(12):1200.

5. Gash KJ, Baser O, Kiran RP. Factors associated with degree of tumour response to neo-adjuvant radiotherapy in rectal cancer and subsequent corresponding outcomes. Eur J Surg Oncol. 2017;43(11):2052-9.

6. Chand M, Yu S, Swift Rl, Brown G. Mucinous carcinoma of the rectum: a distinct clinicopathological entity. Techniques in Coloproctology. 2014;18(4): $335-44$

7. Canda AE, Terzi C, Gorken IB, Oztop I, Sokmen S, Fuzun M. Effects of preoperative chemoradiotherapy on anal sphincter functions and quality of life in rectal cancer patients. Int J Color Dis. 2010;25(2):197-204.

8. Chun HK, Choi D, Kim MJ, Lee J, Yun SH, Kim SH, Lee SJ, Kim CK: Preoperative staging of rectal cancer: comparison of 3-T high-field MRI and endorectal sonography. Ajr Am J Roentgenol 2006, 187(6):1557-1562.

9. Beets-Tan RGH, Beets GL, Vliegen RFA, Kessels AGH, Van Boven H, De Bruine A, von Meyenfeldt MF, Baeten C, van Engelshoven JMA. Accuracy of magnetic resonance imaging in prediction of tumour-free resection margin in rectal cancer surgery. Lancet. 2001:357(9255):497-504.

10. Group MS. Diagnostic accuracy of preoperative magnetic resonance imaging in predicting curative resection of rectal cancer: prospective observational study. Bmj British Medical Journal. 2006;333(7572):779-82.

11. Pham $\Pi$, Liney GP. Wong $K$, Barton MB. Review article: functional MRI for quantitative treatment response prediction in locally advanced rectal Cancer. Br J Radiol. 2017:20151078.

12. Bäuerle T, Seyler L, Münter M, Jensen A, Brand K, Fritzsche KH, KoppSchneider A, Schüssler M, Schlemmer HP, Stieltjes B. Diffusion-weighted imaging in rectal carcinoma patients without and after chemoradiotherapy: a comparative study with histology. Eur J Radiol. 2013;82(3):444-52.

13. Ganten MK, Schuessler M, Bäuerle T, Muenter M, Schlemmer HP, Jensen A, Brand K, Dueck M, Dinkel J, Koppschneider A. The role of perfusion effects in monitoring of chemoradiotherapy of rectal carcinoma using diffusionweighted imaging. Cancer Imaging. 2013;13(4):548.

14. Zhu HB, Zhang XY, Zhou XH, Li XT, Liu YL, Wang S, Sun YS. Assessment of pathological complete response to preoperative chemoradiotherapy by means of multiple mathematical models of diffusion-weighted MRI in locally advanced rectal cancer: a prospective single-center study. J Magn Reson Imaging. 2017;46(1).

15. Le Bihan D: Molecular diffusion, tissue microdynamics and microstructure. NMR Biomed 1995, 8(7-8):375-386.

16. Jensen JH, Helpern JA, Ramani A, Lu H, Kaczynski K. Diffusional kurtosis imaging: the quantification of non-gaussian water diffusion by means of magnetic resonance imaging. Magn Reson Med. 2005:53(6):1432-40.

17. Raab P, Hattingen EK, Zanella FE, Lanfermann H. Cerebral gliomas: diffusional kurtosis imaging analysis of microstructural differences. Radiology. 2010;254(3):876-81.
18. Sun K, Chen X, Chai W, Fei X, Fu C, Yan X, Zhan Y, Chen K, Shen K, Yan F. Breast Cancer: diffusion kurtosis MR imaging-diagnostic accuracy and correlation with clinical-pathologic factors. Radiology. 2015;277(1):46-55.

19. Ma G, Xu XQ, Hu H, Su GY, Shen J, Shi HB, Wu FY. Utility of readoutsegmented Echo-planar imaging-based diffusion kurtosis imaging for differentiating malignant from benign masses in head and neck region. Korean J Radiol. 2018;19(3):443-51.

20. Qi XX, Shi DF, Ren SX, Zhang SY, Li L, Li QC, Guan LM. Histogram analysis of diffusion kurtosis imaging derived maps may distinguish between low and high grade gliomas before surgery. Eur Radiol. 2018;28(4):1748-55.

21. Zhu L, Pan ZL, Ma Q, Yang WJ, Shi HY, Fu CX, Yan X, Du LJ, Yan FH, Zhang $H$. Diffusion kurtosis imaging study of rectal adenocarcinoma associated with histopathologic prognostic factors: preliminary findings. Radiology. 2017;284(1):66-76

22. Bosman F, Carneiro F, Hruban R, Theise N. WHO classification of tumours of the digestive system. Geneva, Switzerland: World Health Organization, 2010

23. DeLong ER, DeLong DM, Clarke-Pearson DL. Comparing the areas under two or more correlated receiver operating characteristic curves: a nonparametric approach. Biometrics. 1988;44(3):837-45.

24. Roethke MC, Kuder TA, Kuru TH, Fenchel M, Hadaschik BA, Laun FB, Schlemmer HP, Stieltjes B. Evaluation of diffusion kurtosis imaging versus standard diffusion imaging for detection and grading of peripheral zone prostate Cancer. Investig Radiol. 2015;50(8):483-9.

25. White NS, McDonald CR, Farid N, Kuperman J, Karow D, Schenker-Ahmed NM, Bartsch H, Rakow-Penner R, Holland D, Shabaik A, et al. Diffusionweighted imaging in Cancer: physical foundations and applications of restriction Spectrum imaging. Cancer Res. 2014;74(17):4638-52.

26. Yu SK, Chand M, Tait DM, Brown G. Magnetic resonance imaging defined mucinous rectal carcinoma is an independent imaging biomarker for poo prognosis and poor response to preoperative chemoradiotherapy. Eur J Cancer. 2014:50(5):920-7.

27. Nasu K, Kuroki Y, Minami M. Diffusion-weighted imaging findings of mucinous carcinoma arising in the ano-rectal region: comparison of apparent diffusion coefficient with that of tubular adenocarcinoma. Jpn J Radiol. 2012:30(2):120-7.

28. Li T, Y Y T, Li L, Lu L, Zhuo Y, Lian J, Xiong Y, Kong D, Li K. Use of diffusion kurtosis imaging and quantitative dynamic contrast-enhanced MRI for the differentiation of breast tumors. J Magn Reson Imaging. 2018, 48(5):13581366

29. Lambregts DMJ, Beets GL, Maas M, Curvo-Semedo L, Kessels AGH, Thywissen T, Beets-Tan RGH. Tumour ADC measurements in rectal cancer: effect of $\mathrm{ROI}$ methods on $\mathrm{ADC}$ values and interobserver variability. Eur Radiol. 2011;21(12):2567-74.

Ready to submit your research? Choose BMC and benefit from:

- fast, convenient online submission

- thorough peer review by experienced researchers in your field

- rapid publication on acceptance

- support for research data, including large and complex data types

- gold Open Access which fosters wider collaboration and increased citations

- maximum visibility for your research: over $100 \mathrm{M}$ website views per year

At $\mathrm{BMC}$, research is always in progress.

Learn more biomedcentral.com/submissions 